\title{
DIMENSION OF DIVERGENCE SET OF THE WAVE EQUATION
}

\author{
SEHEON HAM, HYERIM KO, AND SANGHYUK LEE
}

\begin{abstract}
We consider the Hausdorff dimension of the divergence set on which the pointwise convergence $\lim _{t \rightarrow 0} e^{i t \sqrt{-\Delta}} f(x)=f(x)$ fails when $f \in$ $H^{s}\left(\mathbb{R}^{d}\right)$. We especially prove the conjecture raised by Barceló, Bennett, Carbery and Rogers 1 for $d=3$, and improve the previous results in higher dimensions $d \geq 4$. We also show that a Strichartz type estimate for $f \rightarrow e^{i t \sqrt{-\Delta}} f$ with the measure $d t d \mu(x)$ is essentially equivalent to the estimate for the spherical average of $\widehat{\mu}$ which has been extensively studied for the Falconer distance set problem. The equivalence provides shortcuts to the recent results due to Liu [1] and Rogers [18.
\end{abstract}

\section{INTRODUCTION}

Let $d \geq 1$. We consider the wave operator

$$
e^{i t \sqrt{-\Delta}} f(x)=\frac{1}{(2 \pi)^{d}} \int_{\mathbb{R}^{d}} e^{i(x \cdot \xi+t|\xi|)} \widehat{f}(\xi) d \xi .
$$

In this note we are mainly concerned with the pointwise behavior of $e^{i t \sqrt{-\Delta}} f$ as $t \rightarrow 0$, from which we can deduce the pointwise convergence to the initial data of the solution $u$ to the Cauchy problem:

$$
\partial_{t}^{2} u-\Delta u=0, \quad u(\cdot, 0)=u_{0}, \quad \partial_{t} u(\cdot, 0)=u_{1},
$$

where $\left(u_{0}, u_{1}\right) \in H^{s}\left(\mathbb{R}^{d}\right) \times H^{s-1}\left(\mathbb{R}^{d}\right)$. Here, $H^{s}\left(\mathbb{R}^{d}\right)$ denotes the inhomogeneous Sobolev space of order $s$ which is equipped with the norm $\|f\|_{H^{s}}=\left\|\left(1+|\cdot|^{2}\right)^{\frac{s}{2}} \widehat{f}\right\|_{L^{2}}$.

It is well-known that $e^{i t \sqrt{-\Delta}} f$ converges to $f$ almost everywhere as $t \rightarrow 0$ for all $f \in H^{s}\left(\mathbb{R}^{d}\right)$ if $s>1 / 2$ while the convergence fails in general if $s \leq \frac{1}{2}$. The convergence follows from the maximal estimate

$$
\left\|\sup _{0<t<1}\left|e^{i t \sqrt{-\Delta}} f\right|\right\|_{L^{2}\left(\mathbb{R}^{d}\right)} \lesssim\|f\|_{H^{s}\left(\mathbb{R}^{d}\right)}
$$

for $s>1 / 2$, which is an easy consequence of the Sobolev imbedding and Plancherel's theorem. (See for exmaple [3.) The estimate fails for $s \leq 1 / 2$ (see [20]) and almost everywhere convergenece also fails for $s \leq 1 / 2$. This can be shown using Stein's maximal theorem [19], by which almost everywhere convergence for all $f \in H^{s}$ implies $H^{s}-L^{2, \infty}$ bound on the maximal operator $\sup _{0<t<1}\left|e^{i t \sqrt{-\Delta}} f\right|$. On the other

2010 Mathematics Subject Classification. 42B37.

Key words and phrases. divergence set, fractal Strichartz estimate, spherical average. 
hand, it is not difficult to see the $H^{1 / 2}-L^{2, \infty}$ estimate fails by a construction (see Lemma A.2 in Appendix).

We study a more refined pointwise behavior of $e^{i t \sqrt{-\Delta}} f$ by considering the Hausdorff dimension of the divergence set

$$
\mathfrak{D}_{d}(f):=\left\{x: e^{i t \sqrt{-\Delta}} f(x) \not \rightarrow f(x) \text { as } t \rightarrow 0\right\} .
$$

Dimension of $\mathfrak{D}_{d}(f)$ naturally relates to the regularity exponent $s$ for which $f \in H^{s}$. Almost everywhere convergence only tells the divergence set is of Lebesgue measure zero. However, if the initial datum $f$ possesses an additional regularity, one may expect that the set $\mathfrak{D}_{d}$ has smaller dimension. A natural question is how big the (Hausdorff) dimension of $\mathfrak{D}_{d}$ can be depending the regularity of $f$.

To study the question, let us set

$$
\mathcal{D}_{d}(s):=\sup _{f \in H^{s}\left(\mathbb{R}^{d}\right)} \operatorname{dim}_{H} \mathfrak{D}_{d}(f) .
$$

The problem of determining $\mathcal{D}_{d}(s)$ was first considered by Barceló, Bennett, Carbery and Rogers [1] for the more general dispersive equations and the result was later extended by Lucà and Rogers $\left[13\right.$. Since $\left|e^{i t \sqrt{-\Delta}} f\right| \lesssim\|f\|_{H^{s}}$ for $s>d / 2$, it follows $\mathcal{D}_{d}(s)=0$ for $s>d / 2$. On the other hand, $\mathcal{D}_{d}(s)=d$ if $s \leq 1 / 2$ since the $H^{1 / 2}-L^{2, \infty}$ bound (1.1) generally fails. Thus it is sufficient to consider

$$
1 / 2<s \leq d / 2 \text {. }
$$

So, there is nothing interesting if $d=1$. For $d \geq 2$, the problem is no longer trivial. When $d=2$ Barceló et. al. [1] obtained the optimal result: $\mathcal{D}_{2}(s)=2-2 s$ for $3 / 4<s \leq 1$ and $\mathcal{D}_{2}(s)=4-4 s$ for $1 / 2<s \leq 3 / 4$. In higher dimensions the following was conjectured to be true ([1, Theorem 5.1]).

Conjecture 1.1. For $d \geq 3$,

$$
\mathcal{D}_{d}(s)= \begin{cases}d-2 s, & 1 \leq s \leq \frac{d}{2}, \\ d+2-4 s, & \frac{1}{2}<s \leq 1 .\end{cases}
$$

The lower bound was verified by considering specific functions and measures which show the sharpness of maximal estimate against the measures. As is typical with the problems of similar nature, proving the upper bound is more difficult.

Let $\mathbb{B}^{d}(x, \rho) \subset \mathbb{R}^{d}$ denote the closed ball centered at $x$ of radius $\rho$. For $\alpha \in(0, d]$, we denote by $\mathfrak{M}^{d}(\alpha)$ the collection of non-negative Borel measures $\mu$ supported in $\mathbb{B}^{d}(0,1)$ such that

$$
\mu\left(\mathbb{B}^{d}(x, \rho)\right) \leq C_{\mu} \rho^{\alpha}
$$

for all $x \in \mathbb{R}^{d}$ and $\rho>0$ with a constant $C_{\mu}$. If $\mu \in \mathfrak{M}^{d}(\alpha)$, we define

$$
\langle\mu\rangle_{\alpha}=\sup _{x \in \mathbb{R}^{d}, \rho>0} \rho^{-\alpha} \mu\left(\mathbb{B}^{d}(x, \rho)\right) .
$$

An approach to obtain the upper bound on $\mathcal{D}_{d}(s)$ is to exploit the decay order $\beta=\beta(\alpha)$ of the estimate for $L^{2}$ average of $\widehat{\mu}$ over the sphere:

$$
\int_{\mathbb{S}^{d-1}}|\widehat{\mu}(\lambda \theta)|^{2} d \theta \lesssim \lambda^{-\beta}\|\mu\|\langle\mu\rangle_{\alpha}, \quad \mu \in \mathfrak{M}^{d}(\alpha),
$$

where $\|\mu\|$ is the total variation of $\mu$. 
The estimate (1.3) was previously studied by various authors $([21,7,8])$ being related to the Falconer distance set problem, and further improvements were recently obtained $([13,6])$. These improvements rely on sophisticated argument which has been developed in the study of Fourier restriction problem.

An upper bound on $\mathcal{D}_{d}(s)$ can be obtained using (1.3). In fact, it was shown in [1] that $\mathcal{D}_{d}(s) \leq \alpha$ if (1.3) holds with $\beta>d-2 s$. Thus, making use of the best estimate so far (see [14, 6]), we have, for $d \geq 3$,

$$
\mathcal{D}_{d}(s) \leq \begin{cases}d-2 s, & \frac{d+1}{4}<s \leq \frac{d}{2}, \\ \frac{d^{2}-2 d s}{d-1}, & \frac{1}{2}<s \leq \frac{d+1}{4} .\end{cases}
$$

The bound in the case of $\frac{d+1}{4}<s \leq \frac{d}{2}$ coincides with the sharp upper bound of $\beta=\alpha$ for $0<\alpha \leq \frac{d-1}{2}$ (see [14]). However, when $\frac{1}{2}<s \leq \frac{d+1}{4}$ and $d \geq 4$, the current approach based on (1.3) does not seem to be efficient enough to prove the optimal upper bound for $\mathcal{D}_{d}(s)$. In fact, considering the known upper bound on $\beta$ (for example see [4) for which (1.3) holds, the conjectured optimal upper bound $\mathcal{D}_{d}(s)=d+2-4 s$ which corresponds to the estimate (1.3) with $\beta=\frac{d+\alpha-2}{2}$ can not be achieved by the aforementioned implication from (1.3).

In this paper we take an alternative approach which relies on the fractal Strichartz estimate with respect to a measure (see (1.7) below), which was previously studied by some authors (see $22,17,2,10,18$ ). Via the approach we prove the conjecture (1.2) when $d=3$ and improve the previously known results (see (1.4) ) for higher dimensions $d \geq 4$ and $\frac{1}{2}<s \leq \frac{d+1}{4}$.

Theorem 1.2. Let $d \geq 3$. Then

$$
\mathcal{D}_{d}(s) \leq\left\{\begin{array}{lr}
d-2 s, & \frac{d+1}{4} \leq s \leq \frac{d}{2} \\
\frac{3 d+1}{2}-4 s, & \frac{d}{4} \leq s \leq \frac{d+1}{4} \\
\frac{d^{2}-d-1}{d-2}-\frac{2(d-1) s}{d-2}, & \frac{1}{2}<s \leq \frac{d}{4}
\end{array}\right.
$$

In Figure 1 we compare the result in Theorem 1.2, the conjectured optimal bound (1.2) and the previously known result (1.4).

1.1. Maximal estimates with respect to general measures. To examine the size of the divergence set, we consider the maximal estimate

$$
\left\|\sup _{0<t<1}\left|e^{i t \sqrt{-\Delta}} f\right|\right\|_{L^{2}(d \mu)} \lesssim\langle\mu\rangle_{\alpha}^{\frac{1}{2}}\|f\|_{H^{s}\left(\mathbb{R}^{d}\right)} .
$$

It was shown in [1] that the estimate (1.5) holds only if $s \geq \max \left(\frac{d-\alpha}{2}, \frac{d+2-\alpha}{4}\right)$. (See also Lemma A.1 in Appendix A). In fact, the lower bound of $\mathcal{D}_{d}(s)$ was obtained by proving the failure of (1.5) for $s<\max \left(\frac{d-\alpha}{2}, \frac{d+2-\alpha}{4}\right)$ which is combined with Frostman's lemma.

For $s \in(1 / 2, d / 2]$ let us define $\alpha_{*}(s)$ to be the infimum of $\alpha$ such that the estimate (1.5) holds. Then, by a standard argument (see, for example, [1, Appendix B] or [15. Section 17]), it follows $\mathcal{D}_{d}(s) \leq \alpha_{*}(s)$. Thus Theorem 1.2 is a consequence of the following. 

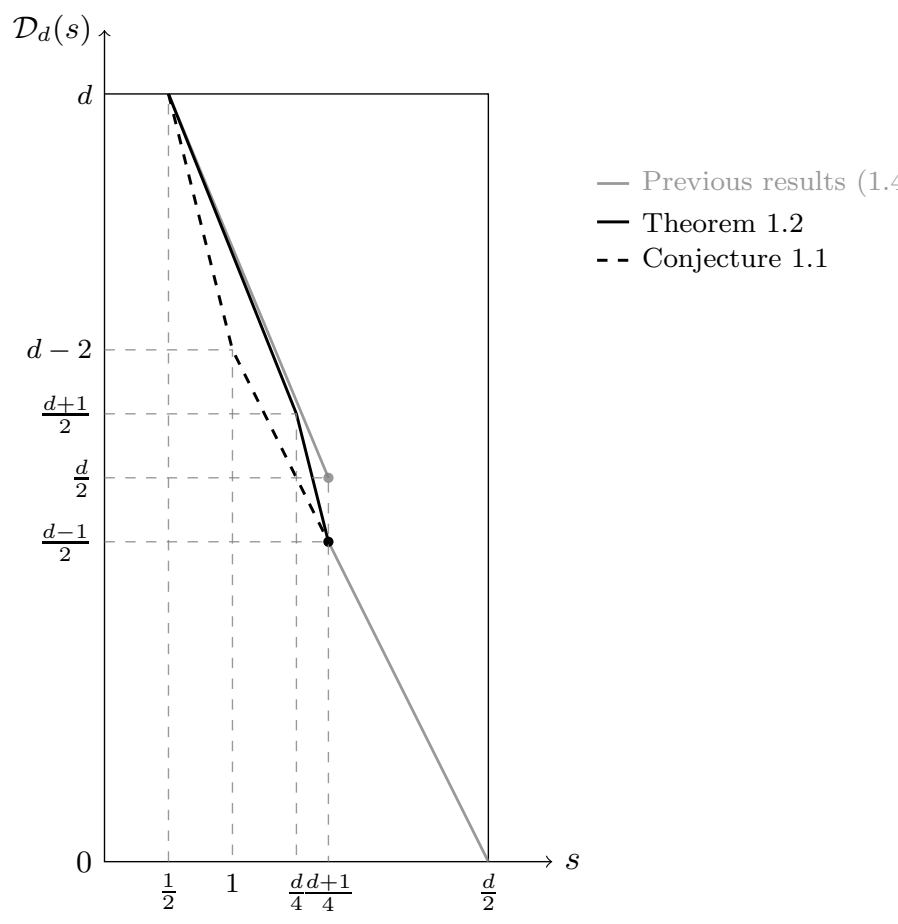

FiguRE 1. Dimension of the divergence set, $d \geq 6$

Theorem 1.3. Let $d \geq 3$ and $\mu \in \mathfrak{M}^{d}(\alpha)$. Then we have (1.5) if

$$
s>s(\alpha, d):=\left\{\begin{array}{lr}
\frac{d-\alpha}{2}, & 0<\alpha \leq \frac{d-1}{2}, \\
\frac{3 d+1}{8}-\frac{\alpha}{4}, & \frac{d-1}{2}<\alpha \leq \frac{d+1}{2} \\
\frac{d-\alpha}{2}+\frac{\alpha-1}{2(d-1)}, & \frac{d+1}{2}<\alpha \leq d .
\end{array}\right.
$$

Especially $s(\alpha, 3)=\frac{5-\alpha}{4}$ for $\alpha \in(1,3]$, which is sharp.

Concerning the proof of Theorem [1.3. our key observation is that the maximal estimates with respect to an $\alpha$-dimensional measure in $\mathbb{R}^{d}$ can be deduced from a family of estimates with respect to $\alpha$-dimensional measures in $\mathbb{R}^{d+1}$. This can be shown by the Kolmogorov-Seliverstov-Plessner linearization argument. Thus the Sobolev exponent $s$ in (1.5) is closely related to the exponent $\gamma=\gamma(\alpha)$ for which the fractal Strichartz estimate

$$
\left\|e^{i t \sqrt{-\Delta}} f\right\|_{L^{2}(d \nu)} \lesssim\langle\nu\rangle_{\alpha}^{\frac{1}{2}}\|f\|_{H^{\gamma}\left(\mathbb{R}^{d}\right)}
$$

holds for any $\nu \in \mathfrak{M}^{d+1}(\alpha)$. For $d \geq 2$, this type of estimates was studied in connection with geometric measure theory (e.g., [22, 7, 16, 17, 2, 10, 18). In particular, the sharp exponent $\gamma$ was established by Wolff [22], Erdogan [7] when $d=2$ and by Cho and two of the authors 2 when $d=3$. We discuss the estimate further in Section 2 before the proof of Theorem 1.2 . 
1.2. $L^{2}$ estimate for $e^{i t \sqrt{-\Delta}}$ with a product measure. The average decay estimate (1.3) has been of interest in relation to not only the Falconer distance set problem but also its refined variant: the pinned distance set problem. We refer the reader to $6,11,9,12,5$ for the most recent progresses related to the distance set problems.

By the argument due to Mattila [14, it can be shown that if $E$ is a Borel set, then

$$
\operatorname{dim} E>\alpha \Rightarrow|\{|x-y|: x, y \in E\}|>0
$$

provided that (1.3) holds and $\alpha+\beta>d$.

On the other hand, Rogers [18 proposed an approach to Falconer's distance set problem which is based on, instead of (1.3), a Strichartz type estimate for $e^{i t \sqrt{-\Delta}} f$ with respect to fractal measures. Using the Riesz representation theorem, he showed that (1.8) holds for $d-1<2 \alpha<d+1$ if the estimate

$$
\left\|e^{i t \sqrt{-\Delta}} P_{\lambda} f\right\|_{L^{2}\left(\mathbb{R}^{d} \times I ; d \mu d t\right)} \lesssim \lambda^{\frac{d-1}{2}-\frac{\gamma}{2}}\|f\|_{L^{2}\left(\mathbb{R}^{d}\right)}, \quad \mu \in \mathfrak{M}^{d}(\alpha)
$$

holds true for $\gamma>d-\alpha$. Here, $I=(1,2)$ and $P_{\lambda}$ is the standard LittlewoodPaley projection operator which is given by $\widehat{P_{\lambda} f}(\xi)=\psi(|\xi| / \lambda) \widehat{f}(\xi)$ where $\psi \epsilon$ $\mathrm{C}_{c}^{\infty}\left(\left(2^{-1}, 2\right)\right)$.

Interestingly the estimates (1.3) and (1.9) turn out to be essentially equivalent to each other.

Theorem 1.4. Let $\lambda \gg 1$ and $\mu \in \mathfrak{M}^{d}(\alpha)$ for $0<\alpha \leq d$. Then (1.9) holds for $\gamma<\beta$ if the estimate (1.3) holds. Conversely, we have the estimate (1.3) with $\beta=\gamma$ if (1.9) holds.

Recently, Liu [11] deduced the similar implication as (1.8) for the pinned distance set: there exists $x \in E$ such that $D_{x}(E)=\{|x-y|: y \in E\}$ has positive Lebesgue measure if the estimate (1.3) holds and $\alpha+\beta>d$. The estimate

$$
\left\|f * \sigma_{t}\right\|_{L^{2}\left(\mathbb{B}^{d}(0,1) \times I ; d \mu d t\right)} \lesssim\|f\|_{H^{-\frac{\beta}{2}+\epsilon}}
$$

played a crucial role in Liu's argument where $\sigma_{t}$ is the normalized surface measure on the sphere $\{x:|x|=t\}$ and $I=(1,2)$. Using an $L^{2}$ identity, he showed that (1.3) implies (1.10) for any $\epsilon>0$. The estimate (1.10) is an easy consequence of (1.9) since $\widehat{d \sigma_{t}}=c_{+} e^{i t|\xi|} a_{+}(t \xi)+c_{-} e^{-i t|\xi|} a_{-}(t \xi)$ with $a_{ \pm}(t \xi)=O\left(|t \xi|^{-\frac{d-1}{2}}\right)$ when $|\xi| \geq 1$. Using Theorem 1.4 and the argument due to Mattila [14], one can deduce the results in Liu [11] and Rogers [18.

\section{Proof of Theorem 1.3 and Theorem 1.4}

To prove Theorem 1.3, we use the fractal Strichartz estimate (1.7). Concerning the Lebesgue measure the estimate (1.7) is well understood but for the general measure $\nu$ the sharp regularity is not known when $d \geq 4$. When $d=2$, Wolff [22] obtained the estimate (1.7) with $\alpha<1$ and the sharp estimates for $\alpha \geq 1$ was settled by Erdoğan [7. Those results were extended to higher dimensions $(d \geq 3)$ by Cho and two of the authors [2, where, especially for $d=3$, the sharp estimate was proved by using the sharp bilinear restriction estimate for the cone. Recently, 
Harris [10] improved the bound when $d \geq 4$ and $\frac{d+1}{2}<\alpha \leq d$. These results can be summarized as follows.

Theorem 2.1 ([2, 10]). Let $d \geq 3$. For $0<\alpha<d+1$, let $\nu \in \mathfrak{M}^{d+1}(\alpha)$. Then (1.7) holds with $\gamma>\gamma(\alpha, d)$ where

$$
\gamma(\alpha, d)=\left\{\begin{array}{lr}
\frac{d-\alpha}{2}, & 0<\alpha \leq \frac{d-1}{2} \\
\frac{3 d+1}{8}-\frac{\alpha}{4}, & \frac{d-1}{2}<\alpha \leq \frac{d+1}{2} \\
\frac{d-\alpha}{2}+\frac{\alpha-1}{2(d-1)}, & \frac{d+1}{2}<\alpha \leq d \\
\frac{d+1-\alpha}{2}, & d<\alpha \leq d+1
\end{array}\right.
$$

Now we prove Theorem 1.3 making use of Theorem 2.1

Proof of Theorem 1.3. In order to prove Theorem 1.3. by Kolmogorov-SeliverstovPlessner linearization argument, it suffices to show that

$$
\left\|e^{i \mathbf{t}(\cdot) \sqrt{-\Delta}} f\right\|_{L^{2}(d \mu)} \leq C\langle\mu\rangle_{\alpha}^{\frac{1}{2}}\|f\|_{H^{s}\left(\mathbb{R}^{d}\right)}
$$

for any measurable function $\mathbf{t}: \mathbb{B}^{d}(0,1) \rightarrow(0,1)$ with $C$ independent of $\mathbf{t}$.

Let us define a linear functional $\ell$ by

$$
\ell(F)=\int F(x, \mathbf{t}(x)) d \mu(x)
$$

for any continuous function $F$ in $\mathrm{C}_{0}\left(\mathbb{R}^{d+1}\right)$. By the Riesz representation theorem it follows that there is a unique Radon measure $\nu$ on $\mathbb{R}^{d+1}$ such that

$$
\ell(F)=\int F(y, s) d \nu(y, s)=\int F(x, \mathbf{t}(x)) d \mu(x) .
$$

Clearly $\nu$ belongs to $\mathfrak{M}^{d+1}(\alpha)$ and $\left.\langle\nu\rangle_{\alpha} \leq\langle\mu\rangle_{\alpha} 1\right]$ Since $\left\|e^{i \mathbf{t}(\cdot) \sqrt{-\Delta}} f\right\|_{L^{2}(d \mu)}=$ $\left\|e^{i t \sqrt{-\Delta}} f\right\|_{L^{2}(d \nu)}$, applying Theorem 2.1, we see that (1.5) holds if (1.6) is satisfied.

We now proceed to prove Theorem 1.4. The relation of (1.3) and (1.9) is basically due to the following lemma.

Lemma 2.2. Let $q \geq 2$ and $\lambda \gg 1$. For $\mu \in \mathfrak{M}^{d}(\alpha)$, suppose that

$$
\left(\int|\widehat{F}|^{q} d \mu\right)^{\frac{1}{q}} \lesssim \lambda^{s}\|F\|_{2}
$$

whenever supp $F \subset \lambda \mathbb{S}^{d-1}+O(1)$. Then we have

$$
\left(\int_{1}^{2} \int|\widehat{G}|^{q} d \mu d t\right)^{\frac{1}{q}} \lesssim \lambda^{s+\frac{1}{2}-\frac{1}{q}}\|G\|_{2}
$$

whenever supp $G \subset \lambda \Gamma^{d}+O(1)$. Here $\Gamma^{d}:=\left\{(\xi,|\xi|) \in \mathbb{R}^{d+1}: 2^{-1} \leq|\xi| \leq 2\right\}$. In particular, when $q=2$, the estimates (2.3) and (2.4) are equivalent.

\footnotetext{
${ }^{1}$ It is clear that $|(x-y, \mathbf{t}(x)-s)| \leq r$ implies $|x-y| \leq r$ and $\nu\left(\mathbb{B}^{d+1}((y, s), r)\right)=\int \chi_{\mathbb{B}^{d+1}((y, s), r)}(x, \mathbf{t}(x)) d \mu(x) \leq \int \chi_{\mathbb{B}^{d}(y, r)}(x) d \mu(x) \leq\langle\mu\rangle_{\alpha} r^{\alpha}$.
} 
Proof. Assume that (2.3) holds. Let $\psi$ be a smooth function such that $\widehat{\psi} \gtrsim 1$ on $[1,2]$ and $\psi$ is supported in $[-1,1]$. Then it follows

$$
\int_{1}^{2} \int|\widehat{G}(x, t)|^{q} d \mu(x) d t \lesssim \iint_{1}^{2}|\widehat{G}(x, t) \widehat{\psi}(t)|^{q} d t d \mu(x) .
$$

We consider the inverse Fourier transform in $t$ and observe that $(\widehat{G}(x, \cdot) \widehat{\psi}(\cdot))^{\vee}(\tau)=$ $\mathcal{F}_{\xi} G(x, \cdot) * \psi(\tau)$. Here $\mathcal{F}_{\xi}$ denotes the Fourier transform in $\xi$, so that $\widehat{G}(x, t)=$ $\int \mathcal{F}_{\xi} G(x, \tau) e^{i t \tau} d \tau$. For each $x$, it is easy to see that $\mathcal{F}_{\xi} G(x, \cdot) * \psi$ is supported in $[\lambda / c, c \lambda]$ for some $c>1$. Thus, by Bernstein's inequality, we obtain

$$
\iint_{1}^{2}|\widehat{G}(x, t) \widehat{\psi}(t)|^{q} d t d \mu(x) \lesssim \lambda^{\left(\frac{1}{2}-\frac{1}{q}\right) q} \int\left(\int_{1}^{2}|\widehat{G}(x, t) \widehat{\psi}(t)|^{2} d t\right)^{\frac{q}{2}} d \mu(x) .
$$

By Plancherel's theorem in $t$ and Minkowski's inequality, we have

$$
\begin{aligned}
\int\left(\int_{1}^{2}|\widehat{G}(x, t) \widehat{\psi}(t)|^{2} d t\right)^{\frac{q}{2}} d \mu(x) & =\int\left(\int\left|\mathcal{F}_{\xi} G(x, \cdot) * \psi(\tau)\right|^{2} d \tau\right)^{\frac{q}{2}} d \mu(x) \\
& \lesssim\left(\int\left(\int\left|\mathcal{F}_{\xi} G(x, \cdot) * \psi(\tau)\right|^{q} d \mu(x)\right)^{\frac{2}{q}} d \tau\right)^{\frac{q}{2}}
\end{aligned}
$$

Note that

$$
\mathcal{F}_{\xi} G(x, \cdot) * \psi(\tau)=\mathcal{F}_{\xi}\left(\int G(\xi, \rho) \psi(\tau-\rho) d \rho\right) .
$$

Since $\psi$ is supported in $[-1,1]$ and $\operatorname{supp} G \subset \lambda \Gamma^{d}+O(1)$, we see that for each fixed $\tau, \int G(\xi, \rho) \psi(\tau-\rho) d \rho$ is supported in the set ||$\xi|-\tau|=O(1)$ and $\tau \sim \lambda$. Thus, by (2.3) it follows that, for each $\tau$,

$$
\int\left|\mathcal{F}_{\xi} G(x, \cdot) * \psi(\tau)\right|^{q} d \mu \lesssim \lambda^{s q}\left\|\int G(\xi, \rho) \psi(\tau-\rho) d \rho\right\|_{L_{\xi}^{2}}^{q} .
$$

Combining this with (2.5), (2.6) and (2.7), by Minkowski's and Young's convolution inequality we have

$\left(\int_{1}^{2} \int|\widehat{G}|^{q} d \mu d t\right)^{\frac{1}{q}} \lesssim \lambda^{s+\frac{1}{2}-\frac{1}{q}}\left(\int\left\|\int G(\xi, \rho) \psi(\tau-\rho) d \rho\right\|_{L_{\xi}^{2}}^{2} d \tau\right)^{\frac{1}{2}} \lesssim \lambda^{s+\frac{1}{2}-\frac{1}{q}}\|G\|_{2}$.

Therefore we get (2.4).

Now let $q=2$. Let $F$ be a function with $\operatorname{supp} F \subset \lambda \mathbb{S}^{d-1}+O(1)$ and consider

$$
G(\xi, \tau)=F(\xi) \chi_{[\lambda, \lambda+1]}(\tau) .
$$

Then, since $\int_{1}^{2}\left|\widehat{\chi_{[\lambda, \lambda+1]}}(t)\right|^{2} d t \gtrsim 1$ independent of $\lambda$, by Fubini's theorem it is clear

$$
\int|\widehat{F}|^{2} d \mu \lesssim \int_{1}^{2} \int|\widehat{G}|^{2} d \mu d t
$$

By (2.4) with $q=2$ and Plancherel's theorem, it follows that

$$
\int|\widehat{F}|^{2} d \mu \lesssim \lambda^{2 s}\|G\|_{2}^{2}=\lambda^{2 s}\|F\|_{2}^{2}
$$

Hence (2.3) and (2.4) are equivalent when $q=2$.

Using this lemma, we prove Theorem 1.4 
Proof of Theorem 1.4. Since $\mu$ has compact support, by duality and the uncertainty principle, one can easily see that (1.3) is equivalent to

$$
\left(\int|\widehat{F}| d \mu\right)^{2} \lesssim \lambda^{d-1-\beta}\|\mu\|\langle\mu\rangle_{\alpha}\|F\|_{2}^{2}
$$

when $\operatorname{supp} F \subset \lambda \mathbb{S}^{d-1}+O(1)$ and $\mu \in \mathfrak{M}^{d}(\alpha)$. By the argument in [1] the estimate can be strengthened to

$$
\|\widehat{F}\|_{L^{2, \infty}(d \nu)} \lesssim \lambda^{(d-1-\beta) / 2}\langle\nu\rangle_{\alpha}^{1 / 2}\|F\|_{2}
$$

for $\nu \in \mathfrak{M}^{d}(\alpha)$. To see this, we consider a measure $d \nu_{E}=\nu(E)^{-1} \chi_{E} d \nu$ for $\nu \in$ $\mathfrak{M}^{d}(\alpha)$ and a Borel set $E$. Then $\nu_{E} \in \mathfrak{M}^{d}(\alpha),\left\|\nu_{E}\right\| \leq 1$, and $\left\langle\nu_{E}\right\rangle_{\alpha} \leq \nu(E)^{-1}\langle\nu\rangle_{\alpha}$. Applying (2.8) with $\nu_{E}$, we see that $\nu(E)^{-1 / 2} \int_{E}|\widehat{F}| d \nu=\nu(E)^{1 / 2} \int_{E}|\widehat{F}| d \nu_{E} \lesssim$ $\lambda^{\frac{d-1-\beta}{2}}\langle\nu\rangle_{\alpha}^{\frac{1}{2}}\|F\|_{2}$. So, we get (2.9) by taking $E=\{x:|\widehat{F}|>\omega\}$.

Interpolating (2.9) with the trivial estimate $\|\widehat{F}\|_{L^{\infty}(d \nu)} \lesssim \lambda^{\frac{d-1}{2}}\|F\|_{2}$ we obtain $L^{2} \rightarrow L^{q}(d \nu)$ estimate. Since $\operatorname{supp} \nu \subset \mathbb{B}^{d}(0,1)$, taking $q$ arbitrarily close to 2 , we get

$$
\int|\widehat{F}|^{2} d \nu \lesssim \lambda^{d-1-\gamma}\langle\nu\rangle_{\alpha}\|F\|_{2}^{2}
$$

for $\gamma<\beta$. By Lemma 2.2, we can see that (2.10) is equivalent to

$$
\int_{1}^{2} \int|\widehat{G}|^{2} d \nu d t \lesssim \lambda^{d-1-\gamma}\langle\nu\rangle_{\alpha}\|G\|_{2}^{2}
$$

where $\operatorname{supp} G \subset \lambda \Gamma^{d}+O(1)$. Let $\varphi \in \mathcal{S}\left(\mathbb{R}^{d}\right)$ such that $\varphi \geq 1$ on $\mathbb{B}^{d}(0,1)$ and $\operatorname{supp} \widehat{\varphi} \subset \mathbb{B}^{d}(0,1)$. Then the space time Fourier transform of $\varphi e^{i t \sqrt{-\Delta}} P_{\lambda} f$ is supported in $\lambda \Gamma^{d}+O(1)$. By (2.11) and Plancherel's theorem we get

$$
\int_{1}^{2} \int\left|\varphi(x) e^{i t \sqrt{-\Delta}} P_{\lambda} f(x)\right|^{2} d \nu d t \lesssim \lambda^{d-1-\gamma}\langle\nu\rangle_{\alpha}\|f\|_{2}^{2},
$$

which gives (1.9) with $\gamma<\beta$ as desired.

To show the converse implication we note that (1.9) implies (2.11). It can be seen easily by Plancherel's theorem and the standard slicing argument decomposing the conic neighborhood into a family of cones. Thus by Lemma 2.2 we have (2.10) which clearly implies $\left|\int \widehat{F} d \mu\right|^{2} \lesssim \lambda^{d-1-\gamma}\|\mu\|\langle\mu\rangle_{\alpha}\|F\|_{2}^{2}$. Therefore by duality and the uncertainty principle we get (1.3) with $\beta=\gamma$.

\section{Appendix A.}

In this section, we discuss necessary conditions for the estimate

$$
\left\|\sup _{0<t<1}\left|e^{i t \sqrt{-\Delta}} f\right|\right\|_{L^{q}(d \mu)} \lesssim\langle\mu\rangle_{\alpha}^{\frac{1}{q}}\|f\|_{H^{s}\left(\mathbb{R}^{d}\right)} .
$$

Lemma A.1. Let $d \geq 3$. The estimate (A.1) holds for all $\mu \in \mathfrak{M}^{d}(\alpha)$ only if

$$
s \geq \begin{cases}\max \left(\frac{d}{2}-\frac{\alpha}{q}, \frac{d+1}{4}\right), & 0<\alpha \leq 1, \\ \max \left(\frac{d}{2}-\frac{\alpha}{q}, \frac{d+1}{4}-\frac{\alpha-1}{2 q}, \frac{d+2-\alpha}{4}, \frac{d-\alpha}{2}\right), & 1<\alpha \leq d .\end{cases}
$$


Proof of Lemma A.1. We first show $s \geq \frac{d}{2}-\frac{\alpha}{q}$. Let $\widehat{f}(\xi)=\chi_{\mathbb{B}^{d}(0, \lambda)}(\xi)$. If we take $t=1 / \lambda$, then we have $\left|e^{i t \sqrt{-\Delta}} f(x)\right| \gtrsim \lambda^{d}$ for $|x| \leq \lambda^{-1}$, which implies $\sup _{0<t<1}\left|e^{i t \sqrt{-\Delta}} f(x)\right| \geq \lambda^{d}$ for $|x| \leq \lambda^{-1}$. We take $d \mu=\chi_{\mathbb{B}^{d}(0,1 / 2)}(x)|x|^{\alpha-d} d x$. Then it is clear that $\langle\mu\rangle_{\alpha} \lesssim 1$. A simple computation gives $\lambda^{d} \lambda^{-\frac{\alpha}{q}} \lesssim \lambda^{s+\frac{d}{2}}$. This shows $s \geq \frac{d}{2}-\frac{\alpha}{q}$.

Next, we show that

$$
s \geq \begin{cases}\frac{d+1}{4}, & 0<\alpha \leq 1, \\ \frac{d+1}{4}-\frac{\alpha-1}{2 q}, & 1<\alpha \leq d .\end{cases}
$$

Let $P=\left\{\xi=\left(\xi_{1}, \xi^{\prime}\right) \in \mathbb{R} \times \mathbb{R}^{d-1}: \lambda \leq \xi_{1} \leq 2 \lambda,\left|\xi^{\prime}\right| \leq \lambda^{1 / 2}\right\}$. Then we consider $f$ which is given by $\widehat{f}=\chi_{P}$. Then the set $\{(\xi,|\xi|): \xi \in P\}$ is contained in a $(d+1)$-dimensional rectangle $Q$ of dimensions $C \lambda \times \underbrace{C \lambda^{1 / 2} \times \cdots \times C \lambda^{1 / 2}}_{d-1 \text { times }} \times C$ for a constant $C>0$. We see that $\left|e^{i t \sqrt{-\Delta}} f\right| \gtrsim|P|$ on the set $Q^{*}=\left\{\left(x_{1}, x^{\prime}, t\right)\right.$ : $\left.\left|x_{1}-t\right| \leq c \lambda^{-1},\left|x^{\prime}\right| \leq c \lambda^{-1 / 2}, t \leq c\right\}$ for a constant $c \leq 1$. It follows that $\left\|\sup _{0<t<1}\left|e^{i t \sqrt{-\Delta}} f(x)\right|\right\|_{L^{q}(\mu)} \gtrsim|P| \mu\left(\operatorname{Proj}_{x}\left(Q^{*}\right)\right)^{1 / q}$. Here, $\operatorname{Proj}_{x}: \mathbb{R}^{d+1} \rightarrow \mathbb{R}^{d}$ is the orthogonal projection which maps $(x, t)$ to $(x, 0)$.

For a given $\alpha$, let $\ell \in[1, d]$ be an integer such that $\ell-1<\alpha \leq \ell$. For a fixed $\ell$, we define a measure

$$
d \mu=\prod_{i=1}^{d-\ell} d \delta\left(x_{d-i+1}\right)\left|x_{\ell}\right|^{\alpha-\ell} d x_{\ell} d x_{\ell-1} \cdots d x_{1},
$$

for the delta measure $\delta$ and $x=\left(x_{1}, \ldots, x_{d}\right)$. Clearly, $\mu \in \mathfrak{M}^{d}(\alpha)$.

Since $\operatorname{Proj}_{x}\left(Q^{*}\right)$ is comparable to a rectangle given by $\left\{\left(x_{1}, x^{\prime}\right) \in \mathbb{R}^{d}: x_{1} \lesssim\right.$ $\left.1,\left|x^{\prime}\right| \leq c \lambda^{-1 / 2}\right\}$ for a sufficiently large $\lambda$, it is easy to show that $\mu\left(\operatorname{Proj}_{x}\left(Q^{*}\right)\right) \gtrsim$ 1 for $0<\alpha \leq 1$ or $\mu\left(\operatorname{Proj}_{x}\left(Q^{*}\right)\right) \gtrsim \lambda^{-(\alpha-1) / 2}$ for $1<\alpha \leq d$. Thus we get $|P| \mu\left(\operatorname{Proj}_{x}\left(Q^{*}\right)\right)^{\frac{1}{q}} \lesssim \lambda^{s}|P|^{\frac{1}{2}}$ from (A.1). This implies (A.3).

The proof of $s \geq \frac{d+2-\alpha}{4}$ can be shown similarly as in [2], so we shall be brief. Let $P, Q$, and $Q^{*}$ as above. Also let $\phi_{P}$ be a Schwartz function supported in $P$. For an integer $N \sim \lambda^{\frac{\alpha-1}{2}}$, we take

$$
\widehat{f}(\xi)=N^{-\frac{1}{2}} \sum_{k=1}^{N} \phi_{P}(\xi) e^{-i v_{k} \cdot \xi^{\prime}}
$$

where $v_{k}$ are the lattice points on $\mathbb{B}^{d-1}(0,1)$ separated by $\sim \lambda^{-\frac{\alpha-1}{2(d-1)}}$. Then we have $\left|e^{i t \sqrt{-\Delta}} f(x)\right| \gtrsim N^{-\frac{1}{2}}|P| \chi_{S}(x, t)$ for $S=\cup_{k=1}^{N}\left(Q^{*}+\left(0, v_{k}, 0\right)\right)$. Let $d \mu=$ $\lambda^{\frac{d-\alpha}{2}} \chi_{\operatorname{Proj}_{x}(S)} d x$, then $\langle\mu\rangle_{\alpha} \lesssim 1$ and $\mu\left(\operatorname{Proj}_{x}(S)\right) \sim 1$. Also, $\|f\|_{2}^{2} \lesssim \sum_{k}\left\|\widehat{f}_{k}\right\|_{2}^{2} \lesssim$ $|P| \sim \lambda^{\frac{d+1}{2}}$. Therefore, we obtain $N^{-\frac{1}{2}} \lambda^{\frac{d+1}{2}} \lesssim\left\|\sup _{0<t<1}\left|e^{i t \sqrt{-\Delta}} f\right|\right\|_{L^{q}(\mu)} \lesssim$ $\|f\|_{H^{s}} \lesssim \lambda^{s} \lambda^{\frac{d+1}{4}}$. Since $N \sim \lambda^{\frac{\alpha-1}{2}}$, we get $s \geq \frac{d+2-\alpha}{4}$. 
In a similar way, we can prove $s \geq \frac{d-\alpha}{2}$. (See also [18] for an alternative proof.) Let $M$ be an integer satisfying $M \sim \lambda^{\alpha}$. Let us set

$$
\widehat{f}(\xi)=M^{-\frac{1}{2}} \sum_{k=1}^{M} \phi_{\mathbb{B}^{d}(0, \lambda)}(\xi) e^{-i \omega_{k} \cdot \xi}
$$

where $\omega_{k}$ are lattice points in $\mathbb{B}^{d}(0,1)$ separated by $\lambda^{-\frac{\alpha}{d}}$. If we choose $t=1 / \lambda$, then $\sup _{0<t<1}\left|e^{i t \sqrt{-\Delta}} f(x, t)\right| \geq M^{-\frac{1}{2}} \lambda^{d} \chi_{T}(x)$, where $T:=\cup_{k=1}^{M} \mathbb{B}^{d}\left(\omega_{k}, \lambda^{-1}\right)$. Let $d \mu:=\lambda^{d-\alpha} \chi_{T}(x) d x$, then $\langle\mu\rangle_{\alpha} \lesssim 1$ and $\mu(T) \sim 1$. We can conclude that $M^{-\frac{1}{2}} \lambda^{d} \lesssim$ $\left\|\sup _{0<t<1} \mid e^{i t \sqrt{-\Delta}} f\right\|_{L^{q}(\mu)} \lesssim\|f\|_{H^{s}} \lesssim \lambda^{s} \lambda^{\frac{d}{2}}$, which gives $s \geq \frac{d-\alpha}{2}$.

The following shows failure of weak type estimate when $s=1 / 2$.

Lemma A.2. Let $q<\infty$ and let $\mu \in \mathfrak{M}^{d}(\alpha)$. Suppose $\|\mu\| \neq 0$, then the estimate $\left\|\sup _{0<t<1}\left|e^{i t \sqrt{-\Delta}} f\right|\right\|_{L^{q, \infty}(d \mu)} \lesssim\langle\mu\rangle_{\alpha}^{\frac{1}{q}}\|f\|_{H^{\frac{1}{2}\left(\mathbb{R}^{d}\right)}}$ fails.

Proof. Since $\|\mu\| \neq 0$, there is an $r>0$ such that $\mu(\{x: r \leq|x| \leq 1\}) \neq 0$. Let $\beta \in$ $C_{c}((1 / 2,2))$ such that $\int \beta(s) s^{\frac{d-1}{2}} d s=1 /(2 \pi)^{\frac{d}{2}}$. Setting $\beta_{N}(\xi)=N^{-\frac{d+1}{2}} \beta(|\xi| / N)$ for $N \geq 1 / r$, we consider

$$
\int e^{i(x \cdot \xi+t|\xi|)} N^{-\frac{d+1}{2}} \beta(|\xi| / N) d \xi=N^{\frac{d-1}{2}} \iint_{\mathbb{S}^{d-1}} e^{i N s x \cdot \theta} d \theta e^{i N t s} \beta(s) s^{d-1} d s .
$$

Since $\int_{\mathbb{S}^{d-1}} e^{i N s x \cdot \theta} d \theta=(2 \pi)^{\frac{d}{2}}(N s|x|)^{\frac{2-d}{2}} J_{\frac{d-2}{2}}(N s|x|)$, using asymptotic expansion of the Bessel function $\left(J_{\frac{d-2}{2}}(s)=C_{ \pm} s^{-\frac{1}{2}} e^{ \pm i s}+O\left(s^{-\frac{3}{2}}\right), s \geq 1\right)$, and taking $t=|x|$, we see

$$
\int e^{i(x \cdot \xi+|x||\xi|)} \beta_{N}(\xi) d \xi=C_{-}|x|^{-\frac{d-1}{2}}+O\left(N^{-1}\right), \quad r \leq|x| \leq 1
$$

provided that $N \geq 1 / r$. Let us consider $f_{L}$ which is given by $\widehat{f_{L}}=\sum_{1 / r \leq 2^{k} \leq L} \beta_{2^{k}}$. Then by A.4 we see that

$$
e^{i|x| \sqrt{-\Delta}} f_{L}(x)=\sum_{1 / r \leq 2^{k} \leq L} C_{-}|x|^{-\frac{d-1}{2}}+O(1) .
$$

Thus, $\sup _{0<t<1}\left|e^{i t \sqrt{-\Delta}} f_{L}(x)\right| \sim \log L$ if $r \leq|x| \leq 1$ and $L \geq 10^{2} / r^{2}$. If we assume the estimate $\left\|\sup _{0<t<1} \mid e^{i t \sqrt{-\Delta}} f\right\|_{L^{q, \infty}(d \mu)} \lesssim\langle\mu\rangle_{\alpha}^{\frac{1}{q}}\|f\|_{H^{s}\left(\mathbb{R}^{d}\right)}$, then it follows that $(\log L) \mu(\{x: r \leq|x| \leq 1\})^{\frac{1}{q}} \lesssim\langle\mu\rangle_{\alpha}^{\frac{1}{q}}(\log L)^{\frac{1}{2}}$ because $\left\|f_{L}\right\|_{H^{\frac{1}{2}}} \sim(\log L)^{\frac{1}{2}}$. This can not be true unless $\mu(\{x: r \leq|x| \leq 1\})=0$. This is a contradiction.

Acknowledgement. This work was supported by the National Research Foundation of Korea (NRF) grant number NRF-2017R1C1B2002959 (Seheon Ham), NRF2019R1A6A3A01092525 (Hyerim Ko), and NRF-2018R1A2B2006298 (Sanghyuk Lee). 


\section{REFERENCES}

[1] J. A. Barceló, J. Bennett, A. Carbery and K. M. Rogers, On the dimension of divergence sets of dispersive equations, Math. Ann. 349 (2011), no. 3, 599-622.

[2] C.-H. Cho, S. Ham and S. Lee, Fractal Strichartz estimate for the wave equation, Nonlinear Anal. 150 (2017), 61-75.

[3] M. Cowling, Pointwise behaviour of solutions to Schrödinger equations. Harmonic Analysis (Cortona, 1982). Lecture Notes in Math., vol. 992, pp. 83-90. Springer, Berlin (1983)

[4] X. Du, Upper bounds for Fourier decay rates of fractal measures, J. London Math. Soc. 102 (2020), 1318-1336.

[5] X. Du, A. Iosevich, Y. Ou, H. Wang, and R. Zhang, An improved result for Falconer's distance set problem in even dimensions, arXiv:2006.06833

[6] X. Du and R. Zhang, Sharp $L^{2}$ estimate of Schrödinger maximal function in higher dimensions, Ann. of Math. 189 (2019), 837-861.

[7] M. B. Erdoğan, A note on the Fourier transform of fractal measures, Math. Res. Lett. 11 (2004), 299-313.

[8] _ A bilinear Fourier extension theorem and applications to the distance set problem, Int. Math. Res. Not., 23 (2005), 1411-1425.

[9] L. Guth, A. Iosevich, Y. Ou, and H. Wang, On Falconer's distance set problem in the plane, Invent. Math. 219 (2020), 779-830.

[10] T. L. J. Harris, Improved decay of conical averages of the Fourier transform, Proc. Amer. Math. Soc. 147 (2019), 4781-4796.

[11] B. Liu, An $L^{2}$ identity and pinned distance problem, Geom. Funct. Anal. 29 (2019), 283-294.

[12] Hausdorff dimension of pinned distance sets and the $L^{2}$ method, Proc. Amer. Math. Soc., 148 (2020), 333-341.

[13] R. Lucà and K. Rogers, Average decay for the Fourier transform of measures with applications, J. Eur. Math. Soc. 21 (2019), 465-506.

[14] P. Mattila, Spherical averages of Fourier transforms of measures with finite energy; dimension of intersections and distance sets, Mathematika, 34 (1987), 207-228.

[15] _ Fourier analysis and Hausdorff dimension, Cambridge University Press, Cambridge, United Kingdom (2015).

[16] D. Oberlin, Packing spheres and fractal Strichartz estimates in $\mathbb{R}^{d}$ for $d \geq 3$, Proc. Amer. Math. Soc., 134 (2006), 3201-3209.

[17] D. Oberlin and R. Oberlin, Spherical means and pinned distance sets, Commun. Korean Math. Soc. 30 (2015), 23-34.

[18] K. Rogers, Falconer's distance set problem via the wave equation, arXiv:1802.01057

[19] E. M. Stein, On limits of sequences of operators, Ann. Math. 74 (1961), 140-170.

[20] B. Walther, Some $L^{p}\left(L^{\infty}\right)$ - and $L^{2}\left(L^{2}\right)$-estimates for oscillatory Fourier transforms, Analysis of divergence (Orono, ME, 1997), 213-231, Appl. Numer. Harmon. Anal., Birkhäuser Boston, Boston, MA, 1999.

[21] T. Wolff, Decay of circular means of fourier transforms of measures, Int. Math. Res. Not. (1999), 547-567.

[22] _ Local smoothing estimates on $L^{p}$ for large p, Geom. Funct. Anal. 10 (2000), 12371288 .

Department of Mathematical Sciences and Rim, Seoul National University, Seoul 08826, Republic of Korea

Email address: seheonham@snu.ac.kr

Email address: kohr@snu.ac.kr

Email address: shklee@snu.ac.kr 